\title{
Minimum Feature Selection for Epileptic Seizure Classification using Wavelet-based Feature Extraction and a Fuzzy Neural Network
}

\author{
Sang-Hong Lee ${ }^{1}$ and Joon S. Lim ${ }^{2, *}$ \\ ${ }^{1}$ Department of Computer Science \& Engineering, Anyang University, Republic of Korea \\ 2 IT College, Gachon University, Republic of Korea
}

Received: 15 Jun. 2013, Revised: 20 Oct. 2013, Accepted: 21 Oct. 2013

Published online: 1 May. 2014

\begin{abstract}
This paper proposes a method that uses a wavelet transform (WT) and a fuzzy neural network to select the minimum number of features for classifying normal signals and epileptic seizure signals from the electroencephalogram (EEG) signals of people with epileptic symptoms and those of healthy people. WT was used to select the minimum number of features by creating detail coefficients and approximation coefficients from EEG signals. 40 initial features were obtained from the created wavelet coefficients using statistical methods, including frequency distributions and the amounts of variability in frequency distributions. We obtained 32 minimum features with the highest accuracy from the 40 initial features by using a non-overlap area distribution measurement method based on a neural network with weighted fuzzy membership functions (NEWFM). NEWFM obtains the bounded sum of weighted fuzzy membership functions (BSWFM) for the 32 minimum features to identify fuzzy membership functions for the 32 features. Using these 32 minimum features as inputs in the NEWFM resulted in a performance sensitivity, specificity, and accuracy of $99.67 \%, 100 \%$, and $99.83 \%$, respectively.
\end{abstract}

Keywords: Electroencephalogram, epileptic seizure, fuzzy neural networks, feature selection, wavelet transform

\section{Introduction}

Electroencephalograms (EEGs) record the electric activity of the cerebral cortex in the brain and are used to measure or diagnose brain diseases $[1,2,3,4,5,6,7]$. EEG monitoring is an important factor when identifying conditions, particularly of patients with epilepsy. Epilepsy involves the repeated occurrence of seizure symptoms, where chronic disease leads to convulsions and disturbances in consciousness, although there is no physical disorder. Researchers are studying artificial intelligence techniques that use EEG signals to detect and diagnose epilepsy $[2,3,4,5,6,7]$. Wavelet transforms (WTs) are a time-frequency analysis method that is used for EEG signal feature extraction before grafting onto fuzzy neural networks in epileptic seizure signal classification $[2,3,4,5][7]$.

Subasi $[2,3,4][7]$ extracted features based on coefficients created using WTs and the extracted features were used as inputs in diverse fuzzy neural networks.
However, the detail coefficients used in Subasi's experiment were wavelet coefficients rather than approximation coefficients $[2,3,4][7]$. The current study investigated a neural network with weighted fuzzy membership functions (NEWFM) [8,9, 10, 11][20, 21,22], which showed excellent performance in forecasting and classification. Normal signals and epileptic seizure signals were classified from the EEG signals used by Subasi [4]. Detail coefficients and approximation coefficients were created from EEG signals using WT. 40 initial features were obtained from the created wavelet coefficients using statistical methods, including frequency distribution and the amounts of variability in the frequency distribution. We selected 32 minimum features with the highest accuracy from the 40 initial features using a non-overlap area distribution measurement method provided by NEWFM. As a result, 16 features were extracted from the 32 minimum features using the approximation coefficients produced in this experiment.

\footnotetext{
*Corresponding author e-mail: jslim@gachon.ac.kr
} 


\section{Overview of Minimum Feature Selection Models for Classifying Epileptic Seizure Signals}

Figure 2.1 shows the process proposed in this paper for selecting the minimum features required for classifying normal signals and epileptic seizure signals. As shown in Figure 2.1, a minimum feature selection model for classifying normal signals and epileptic seizure signals using wavelet coefficients is created from EEG signals by WT. After WT, features are extracted using the frequency distribution and the amounts of variability in the frequency distribution of the wavelet coefficients. The extracted features are used as inputs in the NEWFM.

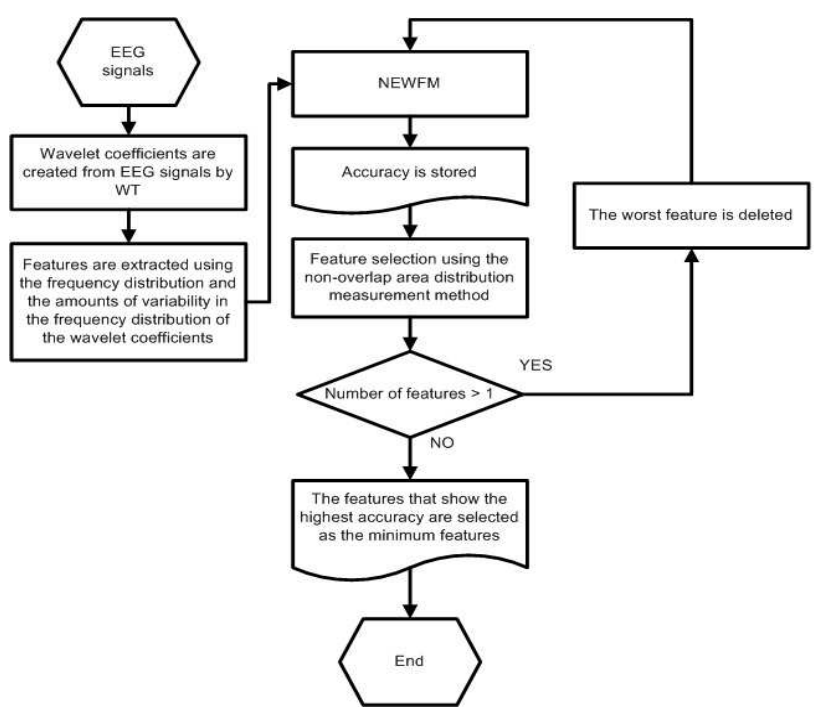

Figure 2.1: Diagram of a minimum feature selection model for classifying normal signals and epileptic seizure signals.

Based on non-overlap area distribution measurement method provided by the NEWFM, the feature that most adversely affects the accuracy is selected and removed. Repeating this feature selection process ultimately selects the features with the highest accuracy as the minimum features for classifying normal signals and epileptic seizure signals.

\subsection{Experimental data}

The EEG signals used by Subasi were used to classify normal signals and epileptic seizure signals [4]. The experimental data used by Subasi were divided into five experimental groups (A, B, C, D, and E) [4][19]. Subasi used experimental groups $\mathrm{A}$ and $\mathrm{E}$ to classify normal signals and epileptic seizure signals. Experimental groups $\mathrm{A}$ and $\mathrm{E}$ were used in this experiment. Experimental group A was composed of normal signals collected from healthy subjects, while experimental group E was composed of epileptic seizure signals collected from subjects with epileptic symptoms. Experimental groups A and E contained 100 files, and each file contained 4097 successive EEG signals. The 4097 successive electroencephalogram signals were divided into 8 sets of 512 signals in total, and the last EEG signal was deleted. Thus, 800 sets of 512 signals were created from the 100 files. The 800 sets were mixed randomly. As shown in Table 1, the first 500 of the 800 sets were used as training sets, while the last 300 sets were used as test sets. The frequency of the experimental data was $173.6 \mathrm{~Hz}$.

Table 1. Numbers of training and test sets

\begin{tabular}{|l|l|l|l|}
\hline Class & Training Set & Test Set & Total Set \\
\hline Epileptic & 500 & 300 & 800 \\
\hline Normal & 500 & 300 & 800 \\
\hline Total & 1000 & 600 & 1600 \\
\hline
\end{tabular}

\subsection{Feature extraction using wavelet transform}

Recent studies use discrete wavelet transforms (DWTs) in signal processing for signal analysis in diverse areas, such as detection of heart disease, forecast of stock prices, and classification of lung sounds [8][17,18]. DWT effects a transformation to basic functions that are localized in scale and in time. DWT decomposes the original signal into a set of coefficients describing the frequency content at given times [12]. In this study, wavelet coefficients with detail coefficients and approximation coefficients at levels 1 to 5 were created from EEG signals by using Haar WT.

Table 2. Feature extraction description

\begin{tabular}{|l|l|}
\hline No & Feature Extraction Description \\
\hline 1 & $\begin{array}{l}\text { Mean of the absolute values of the coefficients in } \\
\text { each sub-band. }\end{array}$ \\
\hline 2 & Median of the coefficients in each sub-band. \\
\hline 3 & $\begin{array}{l}\text { Average power of the wavelet coefficients in each } \\
\text { sub-band. }\end{array}$ \\
\hline 4 & $\begin{array}{l}\text { Standard deviation of the coefficients in each sub- } \\
\text { band. }\end{array}$ \\
\hline 5 & $\begin{array}{l}\text { Ratio of the absolute mean values of adjacent } \\
\text { sub-bands. }\end{array}$ \\
\hline
\end{tabular}

The methods for extracting features from wavelet coefficients are explained in Table 2. Feature extraction methods 1, 3, 4, and 5 are explained in Table 2, and these are the ones used by Subasi [4]. Feature 2 was introduced in our experiments. Features 1, 2, and 3 represent the frequency distributions of the EEG signals, while features 4 and 5 represent the amounts of variability in the frequency distributions [18].

Subasi's experiment used detail coefficients, which are wavelet coefficients rather than approximation coefficients [4]. Detail coefficients and approximation 
coefficients from level 2 to level 5 are explained in Table 2. The feature extraction method was used to extract 20 features from each of the detail coefficients and approximation coefficients. As a result, 40 features were extracted as the initial features.

\subsection{Neural network with weighted fuzzy membership function (NEWFM)}

A neural network with a weighted fuzzy membership function (NEWFM) was used to select the minimum features for classifying normal signals and epileptic seizure signals. The NEWFM has the advantage of providing a non-overlap area distribution measurement method that enables the selection of minimum features. The NEWFM is a supervised classification neuro-fuzzy system that uses the bounded sum of weighted fuzzy membership functions (BSWFMs) $[8,9,10,11]$ [21]. The structure of the NEWFM, as shown in Figure 2.2, is composed of three layers, i.e., the input, hyperbox, and class layers. We extracted 40 initial features from wavelet coefficients using the feature extraction method explained in Table 2, and these initial features were used as inputs in the NEWFM, as shown in Figure 2.2.

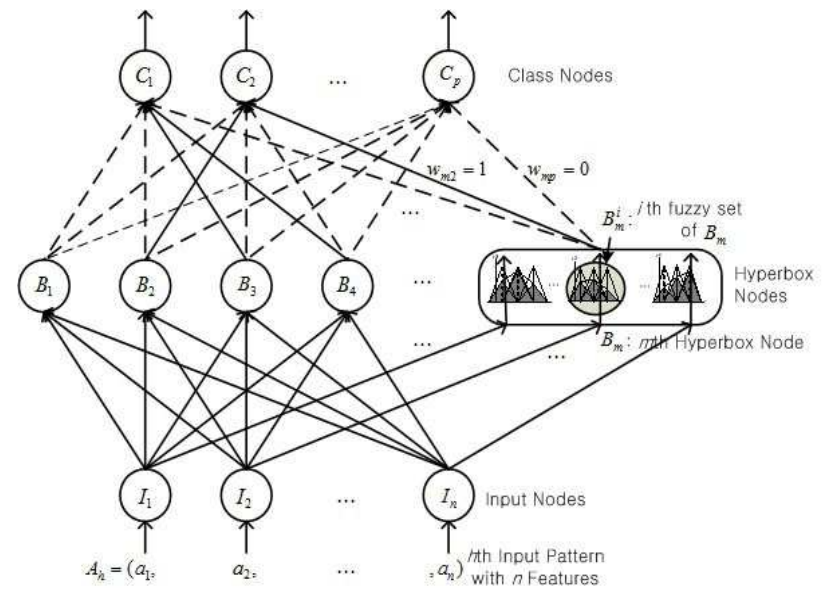

Figure 2.2: Structure of the NEWFM.

\section{Feature Selection}

Feature selection is implemented for better performance, which includes high accuracy and low operation costs using minimum features. Feature selection is an important research area in pattern recognition and machine learning $[13,14,15,16]$. Selecting good features confers advantages such as reduced operation costs or improved performance owing to the removal of redundant or noisy features. To select the best features, we selected 32 minimum features with the highest accuracy from the 40 initial features by using the non-overlap area distribution measurement method provided by NEWFM. This method measures the degree of salience of the ith feature for the non-overlapped areas based on the area distribution, using the following equation [2]:

$$
f(i)=\left(\text { Area }_{E}^{i}+\text { Area }_{N}^{i}\right)^{2} / \frac{1}{\left(1+e^{-\mid \text {Area }_{E}^{i}-\text { Area }_{N}^{i} \mid}\right)}
$$

where Area $_{E}$ and $A r e a_{N}$ are the epileptic seizure superior area and the normal superior area, respectively. The detail coefficient medians at level 5 are shown for Area $_{E}$ and Area $_{N}$ in Figure 3.1. A higher value of $f(i)$ indicates greater importance for the feature characteristic.

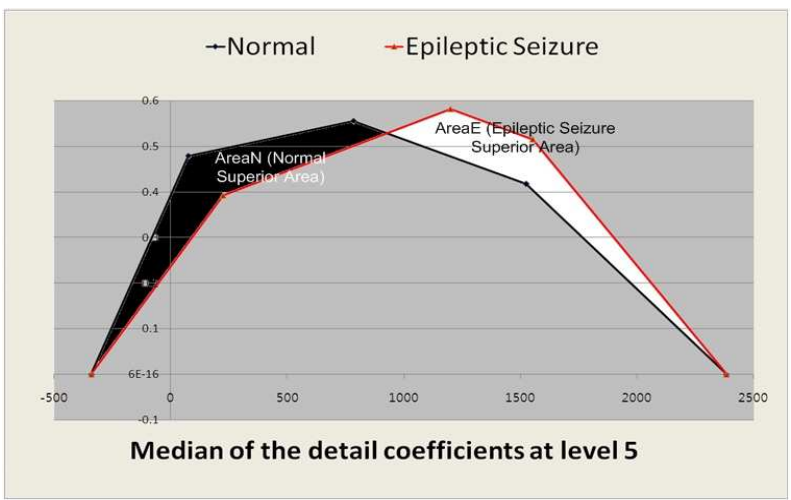

Figure 3.1: Area $_{E}$ (white) and Area $_{N}$ (black) for the detail coefficient medians at level 5.

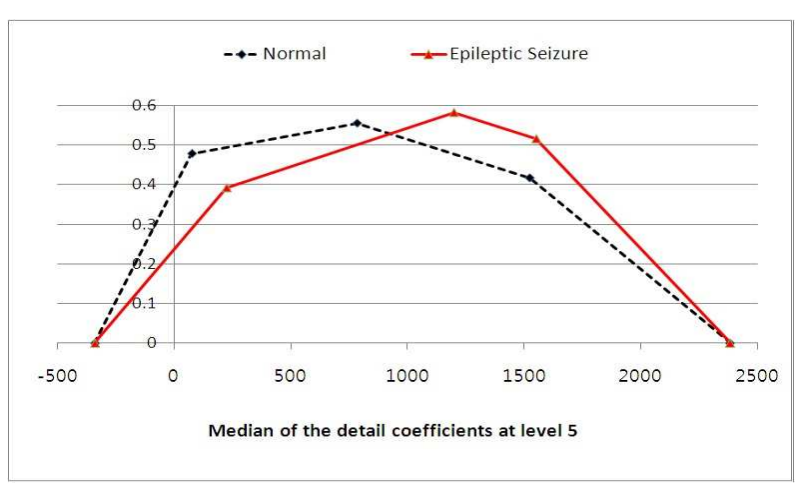

(a) An example of a good candidate feature for feature selection.

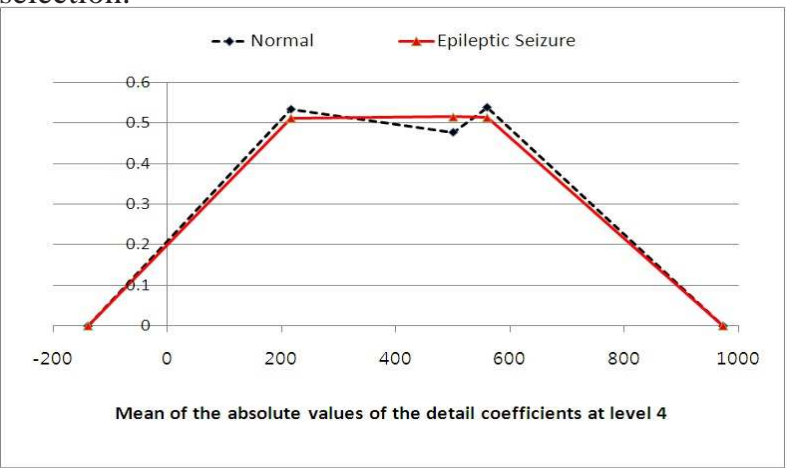

(b) An example of a bad candidate feature for feature selection.

Figure 3.2: Examples of good and bad candidate features for feature selection. 
Table 3. Description of the deleted features

\begin{tabular}{|c|c|c|c|c|c|}
\hline Detail coefficient & No. in Table 2 & Deleted & Approximation coefficient & No. in Table 2 & Deleted \\
\hline \multirow{5}{*}{ Detail coef. at level 2} & 1 & & \multirow{5}{*}{ Approximation coef. at level 2} & 1 & \\
\hline & 2 & & & 2 & \\
\hline & 3 & & & 3 & $\sqrt{ }$ \\
\hline & 4 & & & 4 & \\
\hline & 5 & $\sqrt{ }$ & & 5 & \\
\hline \multirow{5}{*}{ Detail coef. at level 3} & 1 & & \multirow{5}{*}{ Approximation coef. at level 3} & 1 & \\
\hline & 2 & & & 2 & \\
\hline & 3 & & & 3 & $\sqrt{ }$ \\
\hline & 4 & & & 4 & \\
\hline & 5 & $\sqrt{ }$ & & 5 & \\
\hline \multirow{5}{*}{ Detail coef. at level 4} & 1 & & \multirow{5}{*}{ Approximation coef. at level 4} & 1 & \\
\hline & 2 & & & 2 & \\
\hline & 3 & & & 3 & $\sqrt{ }$ \\
\hline & 4 & & & 4 & \\
\hline & 5 & $\sqrt{ }$ & & 5 & \\
\hline \multirow{5}{*}{ Detail coef. at level 5} & 1 & & \multirow{5}{*}{ Approximation coef. at level 5} & 1 & \\
\hline & 2 & & & 2 & \\
\hline & 3 & & & 3 & \\
\hline & 4 & & & 4 & \\
\hline & 5 & $\sqrt{ }$ & & 5 & $\sqrt{ }$ \\
\hline
\end{tabular}

Figure 3.2 shows examples of good and bad candidate features selected from the 40 initial features. The features shown in Figure 3.2 possess two BSWFMs, obtained by the training process in the NEWFM program. The two BSWFMs show the difference between the epileptic seizure signal and the normal signal for each input feature. Therefore, the features shown in Figure 3.2 (b) are in a state where the two BSWFMs almost overlap each other, unlike the features in Figure 3.2 (a) that indicates that these features can be considered ambiguous features that are not easily divided into normal signals and epileptic seizure signals. The value obtained using formula $f(i)$ in Eq. (3.1) of the features shown in Figure 3.2 (a) is greater than that obtained using formula $f(i)$ in Eq. (3.1) of the feature shown in Figure 3.2 (b).

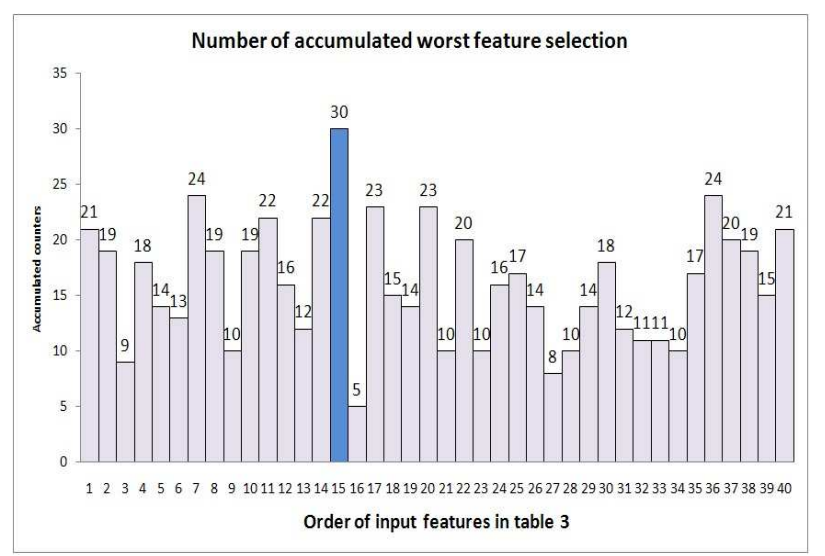

Figure 3.3: Numbers of accumulated features with the smallest non-overlap area for feature selection.

Experiments were conducted with the training and test sets using the 40 initial features shown in Table 1 as inputs of the NEWFM to obtain the highest performance in classifying normal signals and epileptic seizure signals. The training processes and testing processes were iterated 1000 times and the formula $f(i)$ values for individual features were obtained. Features with minimum formula $f(i)$ values were accumulated. Figure 3.3 shows a state where the minimum formula $f(i)$ values were accumulated for all features during 1000 iterations of the experiment. The 15 th feature was judged to be the worst one, and hence, the 15 th feature was deleted before the next experiment started.

Table 3 shows the features deleted from the 40 initial features. As shown in Table 3, the final 32 features selected consisted of 16 detail coefficients and 16 approximation coefficients. Thus, there were good features among the approximation coefficients included in the wavelet coefficients. This result represents a big difference from other studies, which mainly use detail coefficients $[2,4][7]$.

\section{Experimental Results}

Normal signals and epileptic seizure signals were classified with the EEG experimental data used by Subasi [4]. Figure 3.2 (a) and (b) show the bounded sum of the weighted fuzzy membership functions (BSWFM) for 2 feature inputs from the 40 feature inputs. The differences between normal signals and epileptic seizure signals among the 40 feature inputs are evident, and related characteristics can be analyzed.

To assess the performance of the NEWFM, the NEWFM was compared with the mixture-of-experts model (ME) [4], which was Subasi's classification model. The classification performance results are shown in Table 4 and Table 5. TP (True Positive) refers to cases where 
epileptic seizure signals were classified as epileptic seizure signals and TN (True Negative) refers to cases where normal signals were classified as normal signals. FP (False Positive) refers to cases where epileptic seizure signals were classified as normal signals and FN (False Negative) refers to cases where normal signals were classified as epileptic seizure signals. The sensitivity, specificity, and accuracy obtained, as given in Table 5, were determined using the following equations (2), (3), and (4).

$$
\begin{gathered}
\text { Sensitivity }=\frac{T P}{T P+F N} \times 100 \\
\text { Specificity }=\frac{T N}{T N+F P} \times 100 \\
\text { Accuracy }=\frac{T P+T N}{T P+F N+T N+F P} \times 100
\end{gathered}
$$

Table 4. Confusion matrix of classification results

\begin{tabular}{|r|l|l|}
\hline Epileptic seizure signals & TP & FN \\
\cline { 2 - 3 } & 299 & 1 \\
\hline Normal signals & FP & TN \\
\cline { 2 - 3 } & 0 & 300 \\
\hline
\end{tabular}

Table 5. Comparisons of performance results in EEG signal classification for the mixture-of-experts method [4] and NEWFM

\begin{tabular}{|l|l|l|l|}
\hline & Accuracy & Specificity & Sensitivity \\
\hline $\begin{array}{l}\text { Mixture of } \\
\text { expert }\end{array}$ & $94.5 \%$ & $94 \%$ & $95 \%$ \\
\hline NEWFM & $99.83 \%$ & $100 \%$ & $99.67 \%$ \\
\hline
\end{tabular}

\section{Conclusion}

Normal signals and epileptic seizure signals were classified from EEG signals using statistical methods, including frequency distributions and the amounts of variability in frequency distributions, which helped to extract 40 features from wavelet coefficients. We selected 32 minimum features from the 40 extracted features that had the highest accuracy using the non-overlap area distribution measurement method provided by the NEWFM. These 32 minimum features consisted of 16 features extracted from detail coefficients and 16 features extracted from approximation coefficients. These experimental results indicate that some good features were found among the features extracted from approximation coefficients. This represents a major difference from other studies, which mainly use features extracted from detail coefficients. Based on this improved performance, a real-time system for classifying normal signals and epileptic seizure signals can be implemented by measuring EEG signals in real-time using the proposed method. The NEWFM obtained the bounded sum of weighted fuzzy membership functions (BSWFM) for the 32 minimum features and identified the fuzzy membership functions for the 32 features. These fuzzy membership functions were used to classify normal signals and epileptic seizure signals from EEG signals. Using the 32 minimum features as inputs of the NEWFM provided better sensitivity, specificity, and accuracy (99.67\%, 100\%, and $99.83 \%$, respectively) compared with Subasi's results.

\section{Acknowledgement}

This research was supported by MSIP (the Ministry of Science, ICT and Future Planning), Korea, under the IT-CRSP (IT Convergence Research Support Program) (NIPA-2013-H0401-13-1001) supervised by the NIPA (National IT Industry Promotion Agency).

This research was supported by Basic Science Research Program through the National Research Foundation of Korea (NRF) funded by the Ministry of Education, Science and Technology. (NRF-2012R1A1A2044134).

\section{References}

[1] Kiymik M. K., Akin M., and Subasi A., Automatic recognition of alertness level by using wavelet transform and artificial neural network, Journal of Neuroscience Methods, 139, 231-240 (2004).

[2] Subasi A., Epileptic seizure detection using dynamic wavelet network, Expert Systems with Applications, 29, 343-355 (2005).

[3] Subasi A., Application of adaptive neuro-fuzzy inference system for epileptic seizure detection using dynamic wavelet feature extraction, Computation in Biology and Medicine, 37, 227-244 (2007).

[4] Subasi A., EEG signal classification using wavelet feature extraction and a mixture of expert model, Expert Systems with Applications, 32, 1084-1093 (2007).

[5] Adeli H., Zhou Z., and Dadmehr N., Analysis of EEG records in an epileptic patient using wavelet transform, Journal of Neuroscience Methods, 123, 69-87 (2003).

[6] Srinivasan V., Eswaran C., and Sriraam N.H., Approximate Entropy based Epileptic EEG detection using Artificial Neural Networks, IEEE Transactions on Information Technology in Biomedicine, 11, 288-295 (2007).

[7] Subasi A., Automatic detection of epileptic seizure using dynamic fuzzy neural networks, Expert Systems with Applications, 31, 320-328 (2006).

[8] Lim J. S., Finding Features for Real-Time Premature Ventricular Contraction Detection Using a Fuzzy Neural Network System, IEEE TRANSACTIONS ON NEURAL NETWORKS, 20, 522-527 (2009).

[9] Lim J. S., Finding Fuzzy Rules by Neural Network with Weighted Fuzzy Membership Function, International Journal of Fuzzy Logic and Intelligent Systems, 4, 211-216 (2004). 
[10] Lim J. S., Wang D., Kim Y.-S., and Gupta S., A neuro-fuzzy approach for diagnosis of antibody deficiency syndrome, Neurocomputing, 69, 969-974 (2006).

[11] Lim J. S., Ryu T-W, Kim H-J, and Gupta S., Feature Selection for Specific Antibody Deficiency Syndrome by Neural Network with Weighted Fuzzy Membership Functions, LNCS, 3614, 811-820 (2005).

[12] Mallat S., Zero crossings of a wavelet transform, IEEE Trans. Inf. Theory, 37, 1019-1033 (1991).

[13] Hong Y., Kwong S., Chang Y., and Ren Q., Unsupervised feature selection using clustering ensembles and population based incremental learning algorithm, Pattern Recognition, 41, 2742-2756 (2008).

[14] Nguyen M. H. and Torre F. de la, Optimal feature selection for support vector machines, Pattern Recognition, 43, 584591 (2010).

[15] Sotoca J. M. and Pla F., Supervised feature selection by clustering using conditional mutual informationbaseddistances, Pattern Recognition, 43, 2068-2081 (2010).

[16] Lutu P. E. N. and Engelbrecht A. P., A decision rule-based method for feature selection in predictive data mining, Expert Systems with Applications, 37, 602-609 (2010).

[17] Lee S.-H. and Lim J. S., KOSPI time series analysis using neural network with weighted fuzzy membership functions, LNAI, 4953, 53-62 (2008).

[18] Kandaswamy A., Kumar C. S., Ramanathan R. P., Jayaraman S., and Malmurugan N., Neural classification of lung sounds using wavelet coefficients, Computers in Biology and Medicine, 34, 523-537 (2004).

[19] Andrzejak R. G., Lehnertz K., Mormann F., Rieke C., David P., and Elger C. E., Indications of nonlinear deterministic and finite dimensional structures in time series of brain electrical activity: Dependence on recording region and brain state, Physical Review E, 64, 061907 (2001).

[20] Lee S.-H. and Lim J. S., Minimized Stock Forecasting Features Selection by Automatic Feature Extraction Method, Korean Institute of Intelligent Systems, 19, 206-211 (2009).

[21] Lee S.-H. and Lim J. S., Forecasting KOSPI based on a neural network with weighted fuzzy membership functions, Expert Systems with Applications, 38, 4259-4263 (2011).

[22] Lee S.-H. and Lim J. S., Comparison of DBS and levodopa on resting tremor using a fuzzy neural network system, Measurement, 46, 1995-2002 (2013).

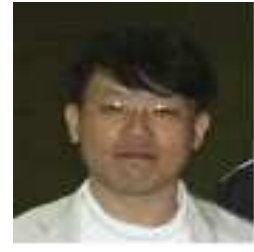

Sang-Hong

Lee received the B.S., M.S., and Ph.D. degrees in computer science from Kyungwon University, Korea in 1999, 2001, and 2012, respectively. $\mathrm{He}$ is currently an assistant professor in the department of computer science \& engineering at Anyang University, Korea. His research focuses on neuro-fuzzy systems, stocks prediction systems, and biomedical prediction systems.

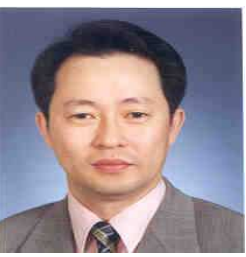

Joon S. Lim received his B.S. and M.S. degrees in computer science from Inha University, Korea, the University of Alabama at Birmingham, and Ph.D. degree was from Louisiana State University, Baton Rouge, Louisiana, in 1986, 1989, and 1994, respectively. He is currently a professor in the department of computer software at Gachon University, Korea. His research focuses on neuro-fuzzy systems, biomedical prediction systems, and human-centered systems. He has authored three textbooks on Artificial Intelligence Programming (Green Press, 2000), Javaquest (Green Press, 2003), and C\# Quest (Green Press, 2006). 